
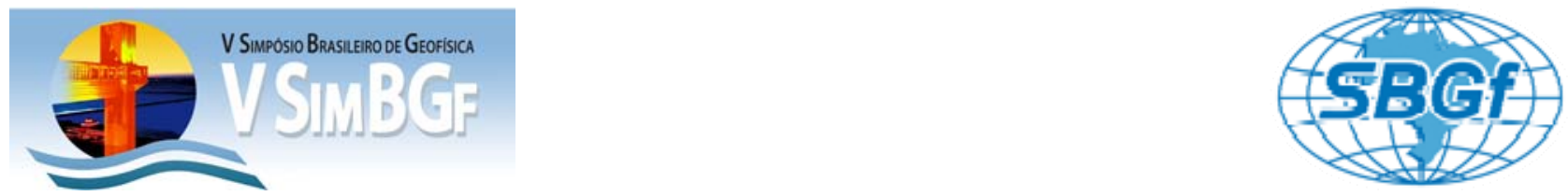

\title{
Estimativa das espessuras das unidades hidrogeológica na região sudeste da cidade de Sinop - MT, a partir de Sondagem Elétrica Vertical.
}

Ricardo Lara dos Santos (IF/UFMT) *; Shozo Shiraiwa (IF/UFMT); Alterêdo Oliveira Cutrim (ICET/UFMT); Frederico Soares Dias (ICET/UFMT) Ivanete Barbosa de Moura (IF/UFMT) Rejane Suellen da Silva Duarte (ICET/UFMT) e Ricardo Cortes Guimarães (ICET/UFMT).

Copyright 2012, SBGf - Sociedade Brasileira de Geofísica

Este texto foi preparado para a apresentação no $V$ Simpósio Brasileiro de Geofísica, Salvador, 27 a 29 de novembro de 2012. Seu conteúdo foi revisado pelo Comitế Técnico do V SimBGf, mas não necessariamente representa a opinião da SBGf ou de seus associados. É proibida a reprodução total ou parcial deste material para propósitos comerciais sem prévia autorização da SBGf

\section{Resumo}

Este estudo foi realizado em uma área localizada no sudeste da cidade. Em Sinop ocorrem os aquíferos Utiariti e Salto das Nuvens. O objetivo do trabalho foi aplicar a técnica da sondagem elétrica vertical (SEV) na estimativa de espessura destes aquíferos. Foram realizadas cinco SEV's utilizando arranjo Schlumberger e abertura $A B / 2$ até $1000 \mathrm{~m}$. Os resultados mostram que o Aquífero Utiariti tem espessura variando de $67 \mathrm{~m}$ a $118 \mathrm{~m}$ e o Salto das Nuvens a espessura superior a $200 \mathrm{~m}$. Estes resultados subsidiarão estudos hidrogeológicos e enriquece o conhecimento da geologia da área.

\section{Introdução}

O conhecimento da profundidade e espessura de unidades hidrogeológicas é de grande importância para o cálculo de reservas e avaliação de vulnerabilidade à contaminação de aquíferos em bacia sedimentar (Cutrim, e Rebouças, 2005; Cutrim, 2009). Em Mato Grosso os sedimentos da Bacia do Parecis vem sendo estudados por Cutrim et al. (2007). Considerando que na área desta pesquisa as águas subterrâneas suprem todas as demandas de água e muito pouco se sabe sobre essas águas, então este trabalho tem como objetivo principal estimar a espessura e a profundidade das unidades hidrogeológicas e a espessura de solo, aplicando o método geofísico de eletrorresistividade utilizando a técnica da sondagem elétrica vertical - SEV.

\section{Geologia e Hidrogeologia da Área}

A área de estudo localiza-se na região centro-norte de Mato Grosso, no município de Sinop e envolve o sudeste da cidade de Sinop -MT e o seu entorno rural (Figura 1). Esta área está inserida na Bacia do Parecis (BARROS et al., 1982), representada pelas formações Utiariti e Salto das Nuvens. A Formação Utiariti é composta por arenitos finos a médio, com estratificação cruzada de pequeno porte e a Formação Salto das Nuvens é constituída por conglomerado polimiticos, arenitos grossos e finos, pelito e argilito, argilito calcífero e marga. Estas formações constituem os principais os aquíferos da área, Utiariti $e$ Salto das Nuvens (RIBEIRO, 2009). Em quase toda extensão da área ocorrem solos do tipo Latossolo Vermelho e Amarelo (PRODEAGRO, 2001).

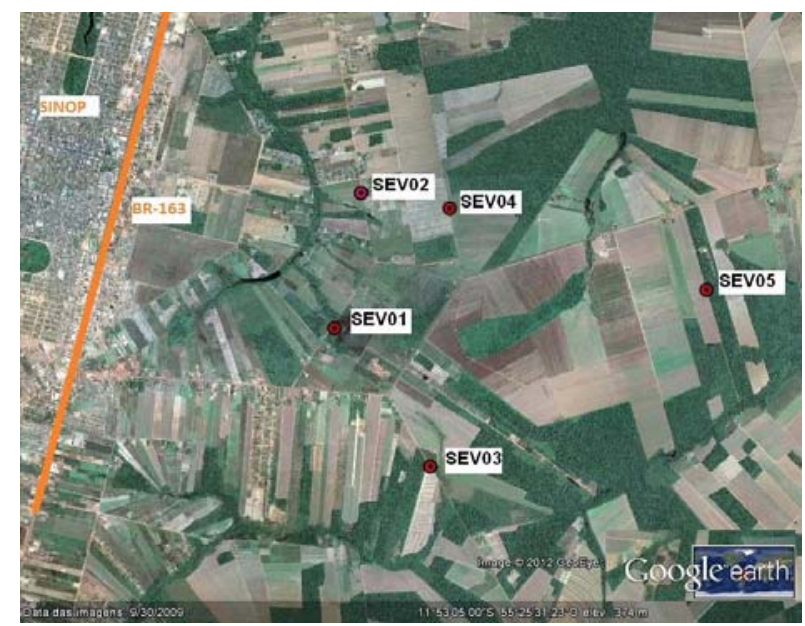

Figura 1. Area de estudo e localização das cinco SEVs, (indicadas em vermelho).

\section{Metodologia/ Problema Investigado}

A técnica da Sondagem Elétrica Vertical - SEV é mundialmente aplicada em estudos de bacias sedimentares na determinação da espessura e profundidade de unidades geológicas. A técnica utilizada com o arranjo Schlumberger (Figura 2), consiste em injetar corrente elétrica no subsolo a partir de dois eletrodos, A e B e medir o potencial em outros dois eletrodos centrais, $\mathrm{M}$ e $\mathrm{N}$.

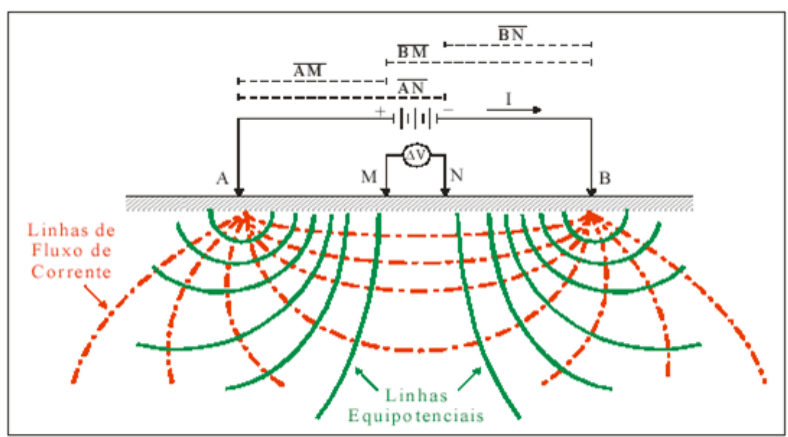

Figura 2- Arranjo eletródico Schlumberger com linhas de Corrente e respectivas equipotenciais (Borges, 2002)

A resistividade aparente é obtida através da medida da diferença de potencial $(\Delta V)$ e da corrente $(I)$ que circula em subsuperfície e do fator geométrico $(K)$ 
Estimativas de espessura de unidades hidrogeológicas na região sudeste da cidade de Sinop - MT, a partir de Sondagem2 Elétrica Vertical (SEV).

aplicando a Equação (1) proposta por Bhattacharya \& Patra (1968):

$$
\rho_{a}=\left(\frac{\Delta V}{I}\right) *\left[2 \pi \cdot\left(\frac{1}{A M}-\frac{1}{B M}-\frac{1}{A N}+\frac{1}{B N}\right)^{-1}\right]
$$

As SEVs foram modeladas utilizando o Software IPI2Win, através do método Ridge Regression (TIKHOOV e ARSENIN, 1997). Foram realizadas cinco SEVs com abertura máxima de $A B / 2$ de $1.000 \mathrm{~m}$, usando um eletrorresistivímetro com potência máxima de $250 \mathrm{~W}$ e voltagem máxima de $800 \mathrm{~V}$. Nesta etapa foram estimadas a resistividade e a espessura das formações geológicas e do solo.

\section{Resultados}

Foram realizadas cinco SEV's utilizando arranjo Schlumberger e abertura $A B / 2$ até $1000 \mathrm{~m}$. A localização das SEVs encontram-se na Figura 1. O comportamento das resistividades elétricas aparente obtido para as SEV's estão apresentados na Figura 3.

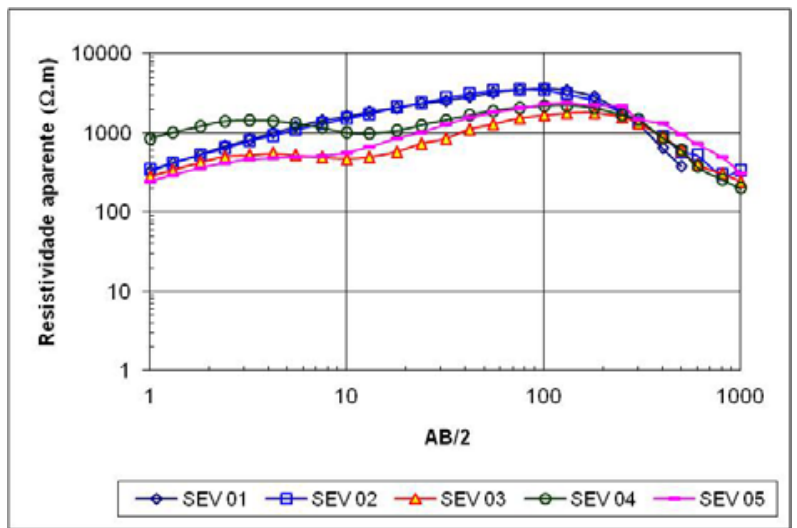

Figura 3 - Curvas de Resistividade aparente em função da distância $A B / 2$ com os dados das Sondagens Elétricas Verticais.

No eixo das ordenadas estão representados os valores das resistividades elétricas aparentes obtidas em campo e no eixo das abscissas a distância entre os eletrodos de corrente $A B / 2$. As curvas estão plotadas em escala logarítmica. As Sev's estão apresentadas em diferentes cores para melhor visualização e interpretação.

Cada uma destas curvas de resistividade aparente, em função de $A B / 2$ foram ajustadas por modelos de camadas de diferentes espessuras e respectivas resistividades elétricas. A Figura 3 mostra o modelo obtido para a SEV 4, ajustado com 7 camadas, sendo as quatro primeiras com resistividade aparente variando de $242 \Omega$.m a $2082 \Omega . m$ com espessura total estimada em torno de 5,69 m correspondente a cobertura pedológica. As diferentes resistividades sugerem variações de umidade e de material pedológico. A quinta e sexta camada possuem resistividades aparente variando de

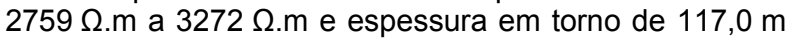

está relacionada a $\mathrm{Fm}$. Utiariti cujo profundidade ao topo é de $5,69 \mathrm{~m}$. A sétima camada apresenta resistividade

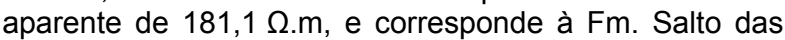
Nuvens, cuja profundidade ao topo foi estimada em $117,0 \mathrm{~m}$ e apresenta espessura superior a investigação teórica de $500 \mathrm{~m}$
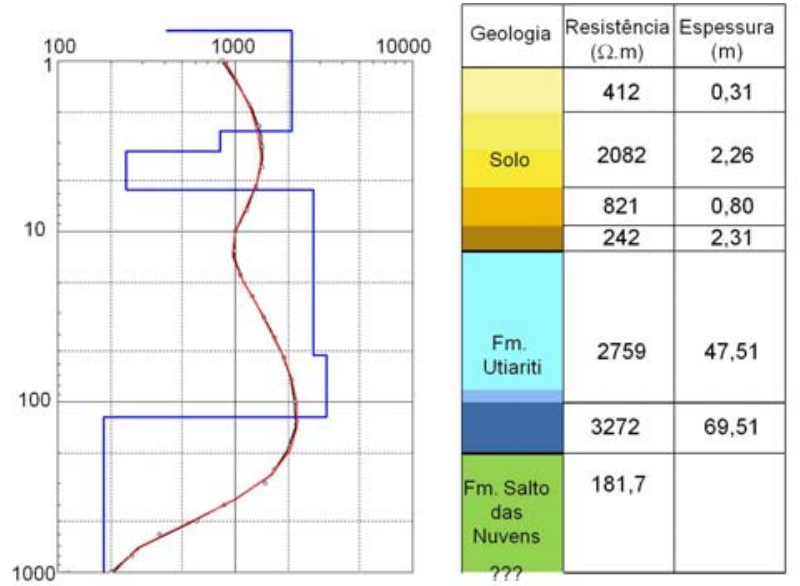

- Modelo de camadas Curva de resistividade aparente:

$$
\text { C Calculada }
$$

Figura 4 - Modelo de camadas ajustadas para a SEV04. No eixo horizontal valores de resistividade (Ohm.m) e no eixo vertical $A B / 2$ profundidade $(m)$.

O mesmo processo de modelagem foi realizado para as demais SEVs e os resultado estão resumidos na TABELA 1.

Observa-se nesta Tabela que as primeiras camadas correspondem ao solo com as respectivas espessuras. A Figura 5 mostra o mapa de da espessura do solo na área de estudo, e verifica-se que ela é maior na região central. e está variando de $3,2 \mathrm{~m}$ a 6,1 m.

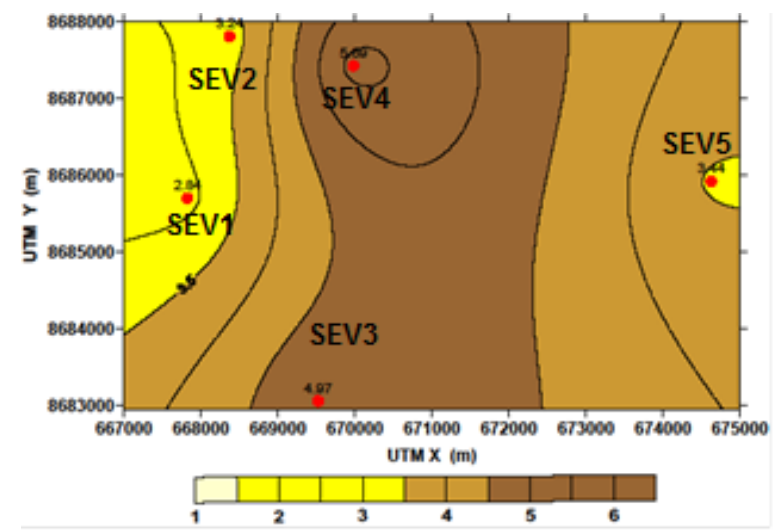

Figura 5 - Mapa de espessura (m) do solo.

A Fm. Utiariti, é o principal aquífero, e sua espessura varia de $67 \mathrm{~m}$ a $118 \mathrm{~m}$. Observa-se que a a maior espessura está localizada na região norte e a menor a noroeste da área Figura 6. 


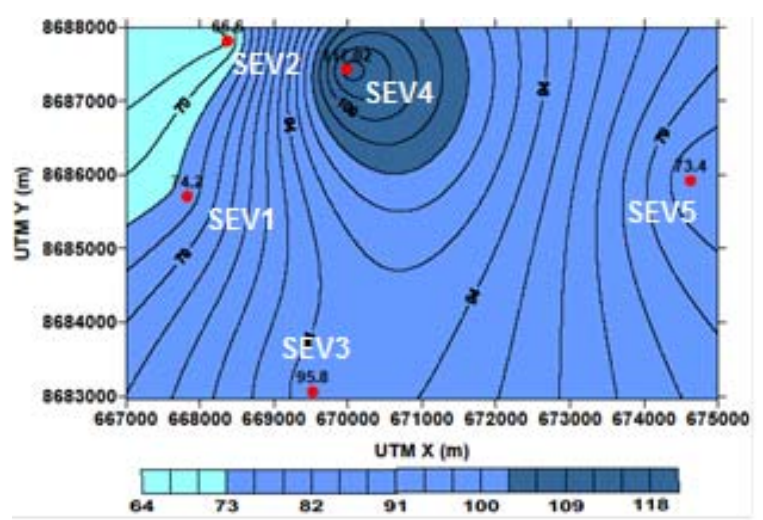

Figura 6 - Mapas de espessura (m) da Formação Utiariti.
$\mathrm{Na}$ Tabela 1, estão representadas a modelagem $\mathrm{e}$ interpretação das cinco SEVs. As camadas representadas em matizes do amarelo estão indicando os valores das resistividades referentes ao solo. Pode-se observar que apenas na SEV4 o solo foi ajustada com quatro camadas. Os valores de resistividade aparente

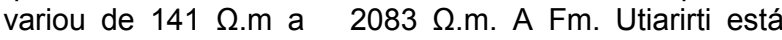
representada em azul e apresentou valores de resistividade variando entre $2759 \Omega$.m a $6585 \Omega$.m. Esta elevação de resistividade ocorre em função da profundidade e indica que granulometria cresce do topo para a base. A Fm. Salto das Nuvens, representada pela cor verde,apresentou resistividade elétrica mais baixa

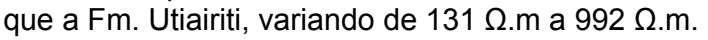

Tabela 1 Espessura, profundidade, resistividade e coordenadas produzidas pela modelagem das SEVs.

\begin{tabular}{|c|c|c|c|c|c|c|c|c|c|c|}
\hline \multicolumn{2}{|c|}{ SEV1 } & \multicolumn{2}{|c|}{ SEV2 } & \multicolumn{2}{|c|}{ SEV3 } & \multicolumn{2}{|c|}{ SEV4 } & \multicolumn{2}{|c|}{ SEV5 } & \multirow[b]{2}{*}{ Geologla } \\
\hline Esp. (m) & $\begin{array}{l}\text { Resist. } \\
\text { ( } \Omega . \mathrm{m})\end{array}$ & Esp. (m) & 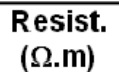 & Esp. (m) & $\begin{array}{l}\text { Resist. } \\
\text { ( } \Omega . \mathrm{m})\end{array}$ & Esp. (m) & $\begin{array}{l}\text { Resist. } \\
(\Omega . m)\end{array}$ & Esp. (m) & $\begin{array}{c}\text { Resist. } \\
\text { ( } \Omega . \mathrm{m})\end{array}$ & \\
\hline 0,5 & 203 & 0,7 & 253 & 0,7 & 217 & 0,3 & 412 & 0,5 & 141 & \\
\hline 3 & 2082 & 1,3 & 2083 & 1,2 & 1506 & 2,3 & 2082 & 0,9 & 1558 & Solo \\
\hline 0,5 & 1186 & 1,2 & 872 & 3 & 136 & 1 & 821 & 2,1 & 156 & \\
\hline 23 & 3135 & 17 & 3750 & 38 & 3056 & 2,5 & 207 & 25 & 3625 & Fm. Utiariti \\
\hline 51 & 6585 & 50 & 5142 & 52 & 3783 & 48 & 2759 & 49 & 4119 & \\
\hline 59 & 490 & 144 & 992 & 107 & 131 & 70 & 3272 & & 983 & $\begin{array}{c}\text { Fm. Salto das } \\
\text { Nuvens }\end{array}$ \\
\hline & 165 & & 241 & & 245 & & 182 & & 214 & \\
\hline
\end{tabular}

\section{Discussão e Conclusões}

As SEV's permitiram estimar a espessura do solo que variou de $3,2 \mathrm{~m}$ a $6,1 \mathrm{~m}$ e a espessura da Formação Utiariti de $66 \mathrm{~m}$ a $117 \mathrm{~m}$. Em nenhuma das SEVs foi possível identificar a base da formação Salto das Nuvens. Os resultados obtidos contribuem para o conhecimento geológico da área e para pesquisa hidrogeológica.

\section{Agradecimentos}

Os autores agradecem ao FINEP pelo financiamento do projeto, e ao CNPQ pela bolsa PIBIC cedida a Ricardo Lara dos Santos

\section{Referências Bibliográficas}

BARROS, L.C., CARDOSO, O.R.F.A., FREIRE, F.A., SOUZA JÚNIOR, J.J., RIVETTI LUZ, D.S., PALMEIRA, R.C.B. \& TASSINARI, C.C.G., 1982. Geologia da folha SD-21. Cuiabá. Projeto RADAMBRASIL, Rio de Janeiro, RJ, 531p.

BHATTACHARYA P.K; PATRA H.P., 1985 Direct Current Geoelectric Sounding. Elsevier Publishing Company, New York, $134 \mathrm{p}$.

BORGES, W. R. 2002. Investigações Geofísicas Na Borda da Bacia Sedimentar de São Paulo, utilizando-se
GPR e eletrorresistividade. Dissertação de Mestrado, apresentado ao Departamento de Geofísica, Universidade de São Paulo - USP.

BRAGA, A.C.O Métodos Geoelétricos Aplicados, 2006. Instituto de Geociências e Ciências Exatas IGCE,Universidade de São Paulo - UNESP.

CUTRIM, A.O. e REBOUÇAS, A.C., 2005. Aplicação de sondagem elétrica vertical na estimativa do topo e da espessura de unidade geológicas da Bacia do Paraná na cidade de Rondonópolis-MT. Revista Brasileira de Geofísica, Rio de Janeiro, v. 23, n. 1, p. 89-98.

CUTRIM, A.O., RUIZ, A.S.; LIPORINI, L.M., MEDEIROS, F.A., BARROSO, U.C., E NASCIMENTO, A.L., 2007. Sondagem elétrica vertical aplicada em pesquisa hidrogeológica na Bacia do Parecís - MT. Revista Brasileira de Geofísica. Rio de Janeiro, v.25 (2).

PRODEAGRO. Programa de Desenvolvimento do Agronegócio, 2001. Aspectos geológicos da Folha Rio Claro - MIR 356 (SD.21-X-C). 235 p.

RIBEIRO, D.B.S., 2009. Avaliação hidrogeológica na cidade de Sinop - MT. Trabalho de Conclusão de Curso (Bacharelado em Geologia). Instituto de Ciências Exatas e da Terra. Departamento de Geologia Geral. Universidade Federal de Mato Grosso, Cuiabá.

TIKNHONOV NA e ARSENIN VY.1997. Solutions of illposed problems. Winston e Sons, New York, 349p. 